\title{
Self-increased acceptance bandwidth of second harmonic generation for high-energy light sources
}

\author{
K.Krupa $^{1, *}$, R. Fona ${ }^{2}$, A. Tonello ${ }^{1}$, A. Labruyère ${ }^{1}$, B. M. Shalaby ${ }^{1,3}$, S. Wabnitz ${ }^{2}$, V. Couderc ${ }^{1}$ \\ ${ }^{1}$ Université de Limoges, XLIM, UMR CNRS 7252, 123 Avenue A. Thomas, 87000 Limoges, France \\ ${ }^{2}$ Dipartimento di Ingegneria dell'Informazione, Università di Brescia, via Branze 38, 25123 Brescia, Italy \\ ${ }^{3}$ Physics Department, Faculty of Science, Tanta University, Tanta, Egypt \\ *Email: katarzyna.krupa@xlim.fr
}

\begin{abstract}
We report a series of experimental results showing that the acceptance bandwidth of second harmonic generation in quadratic crystals may substantially broaden when using pumps at high intensities. We highlight the co-existence of second harmonic generation and strong unconventional spatial beam reshaping. Our experimental results are in good agreement with our numerical simulations.
\end{abstract}

Keywords - nonlinear optics; parametric processes; harmonic generation and mixing; nonlinear optical materials

\section{INTRODUCTION}

Recently the spatiotemporal dynamics of beam propagation has attracted a great interest in the new perspective of generation and manipulation of broadband multicolor light. Nonlinear parametric and scattering effects may change their dynamics when the spatial transverse dimension of the beam is modified along the propagation by the nonlinear material response. In a recent work, Wright and co-authors reported supercontinuum generation in the presence of spatial selffocusing and multiple filamentation occurring in graded-index multimode fibers [1]. Polychromatic spatial beam reshaping has recently been also experimentally demonstrated in periodic crystal structures of waveguide arrays with slow photorefractive or cubic (Kerr) nonlinearities [2].

In this work we focus our attention on the high-intensity nonlinear interplay between fundamental and generated second harmonic $(\mathrm{SH})$ beams in bulk non-centrosymmetric crystals with instantaneous quadratic nonlinearity. We experimentally demonstrate that the acceptance bandwidth of second harmonic generation (SHG) can be significantly increased at high intensities of the pump, as predicted by the analytical theory of two-wave mixing [3]. We show that strong nonlinear coupling between fundamental and its $\mathrm{SH}$ spectral component is also associated with a spatial beam reshaping. Such effect may find potential applications in new concepts of high-energy laser sources, which are required for numerous high-power applications including remote sensing and CARS spectroscopy. Maximum conversion efficiency for SHG is generally achieved when the linear phase-mismatch $(\Delta \mathrm{k})$ among the interacting wave-vectors is zero (phase-matching) or zero on average in a periodic medium (quasi-phase-matching or QPM). Moving away from linear phase matching and in the low-conversion regime, the efficiency drops rapidly, as described by the usual form of a sinc function for the SH field [4].

\section{EXPERIMENTAL SET-UP}

Our experiments were carried out with a high-energy 30ps $20 \mathrm{~Hz}$ Q-switched mode-locked Nd:YAG laser pump at $1064 \mathrm{~nm}$. The pump beam was focused at the input face of the crystal. Its input waist was adjusted to keep nearly the same beam diameter all along the propagation in the crystal when operating in the linear regime (i.e. at very weak intensities of the pump): the diffraction length $\left(\mathrm{L}_{\mathrm{D}}\right)$ was approximately equal to or larger than the physical length of the crystals $(\mathrm{L})$, that is $\mathrm{L}_{\mathrm{D}} \geq \mathrm{L}$. Note that this is not the usual experimental condition, where instead $\mathrm{L}_{\mathrm{D}}<<\mathrm{L}$. We considered different values of phasemismatch for SHG, as well as different input intensities to explore the different interplays between spatial beam reshaping effects and SHG. We used an energy meter and a CCD camera to measure the energy of SHG and the spatial shape of the beam at the output face of the crystals, respectively.

We studied the strong nonlinear regime of SHG in two types of crystals. The first crystal was a $15 \mathrm{~mm}$ long sample of periodically-poled lithium niobate (PPLN), permitting a type- 0 (all waves extraordinarily polarized) QPM for SHG. The second was a $30 \mathrm{~mm}$ long potassium titanyl phosphate (KTP) crystal, where we exploited the crystal anisotropy to achieve type II SHG (the pump is equally divided into ordinary and extraordinary axis, and the $\mathrm{SH}$ is polarized along the extraordinary axis). The value of phase-mismatch for SHG $(\Delta \mathrm{k})$ was controlled by tuning either the crystal temperature, in the case of PPLN, or the input beam external angle, in the case of KTP, when adjusting the pump polarization state.

\section{EXPERIMENTAL RESULTS}

For pump intensities slightly above the linear regime of propagation, we observed standard SHG [4]: the energy of the SHG was maximized at the phase-matching point, with a very narrow acceptance bandwidth, as shown by the black curves of Fig.1a and Fig.2a for PPLN and KTP crystal, respectively. Frequency conversion to the second harmonic was accompanied by the known effect of spatial self-defocusing (self-focusing) of the fundamental wave for negative (positive) values of $\Delta \mathrm{kL}$ : the black curves of Fig.1b and Fig.2b show the corresponding variation of the output fundamental beam diameter upon $\Delta \mathrm{kL}$ for the two crystals $[5,6]$. 
When increasing the pump intensity the SHG grows larger. However, the SHG energy normalized to its maximum level shows that the acceptance bandwidth (variation of $\Delta \mathrm{kL}$, i.e. temperature or angle) increases with the pump intensity [3]. At the same time, the output beam diameter of the fundamental wave is also modified. The experimental results, obtained at high pump intensities, are indicated by the red curves in Fig.1 and Fig. 2 for PPLN and KTP, respectively. In each figure, the phase-mismatch dependence of the normalized energy of the $\mathrm{SH}$ is illustrated in panel a, while the evolution of the corresponding output fundamental beam diameter in presented in panel b. By comparing those figures, we can see that the SHG in PPLN exhibits an asymmetric behavior with respect to $\Delta \mathrm{kL}$ : higher energy values of SHG were observed with nonlinear self-focusing of the pump. In KTP instead, we noticed a more symmetrical broadening of the SHG acceptance bandwidth. Note that the self-defocusing observed at moderate energies for negative values of $\Delta \mathrm{kL}$, leads to an intensity dependent self-focusing effect, which results in the formation of a self-confined beam of a smaller diameter. The choice of a relatively large input beam diameter $\left(\mathrm{L}_{\mathrm{D}} \sim \mathrm{L}\right)$ leads to a beam dynamics and interplay with SHG that are completely different from the situation leading to spatial soliton, which is so far the most studied case $[7,8]$.
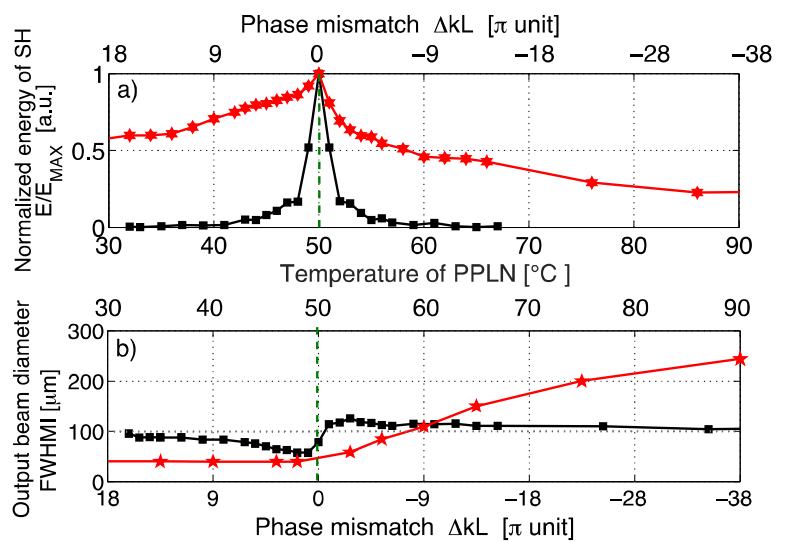

Fig.1. Experimental results of SHG (a) and evolution of output fundamental beam diameter (b) as a function of phase-mismatch $(\Delta \mathrm{kL})$, i.e. temperature for PPLN crystal at low of $15 \mathrm{MW} / \mathrm{cm}^{2}$ (black curve) and high of $5.5 \mathrm{GW} / \mathrm{cm}^{2}$ (red curve) pump intensities; phase-matching temperature for $\mathrm{SHG}$ is at $50^{\circ} \mathrm{C}$ (green dashed line)

\section{NUMERICAL SIMULATIONS}

The main features, which are observed in our experiments were modeled with the coupled mode equations governing the SHG process (see for instance Ref.[9]). For the type-II interaction in KTP, the complex envelopes of the two polarization components $\mathrm{A}(\mathrm{x}, \mathrm{y}, \mathrm{z}), \mathrm{B}(\mathrm{x}, \mathrm{y}, \mathrm{z})$ of the electric field at the fundamental frequency $\omega_{F}$ and the complex envelope $\mathrm{S}(\mathrm{x}, \mathrm{y}, \mathrm{z})$ of the second harmonic $\omega_{S H}$ are governed (in the paraxial approximation and neglecting the walk-off effect) by the following set of nonlinearly coupled equations:

$$
\begin{aligned}
& i 2 k_{A} \frac{\partial A}{\partial z}+\nabla_{\perp}^{2} A+2 \frac{\omega_{F}^{2}}{c^{2}} d_{e f f} S B^{*} e^{-i \Delta k z}=0 \\
& i 2 k_{B} \frac{\partial B}{\partial z}+\nabla_{\perp}^{2} B+2 \frac{\omega_{F}^{2}}{c^{2}} d_{e f f} S A^{*} e^{-i \Delta k z}=0 \\
& i 2 k_{S} \frac{\partial S}{\partial z}+\nabla_{\perp}^{2} S+2 \frac{\omega_{S H}^{2}}{c^{2}} d_{e f f} A B e^{i \Delta k z}=0
\end{aligned}
$$

where $\mathrm{k}_{\mathrm{A}}, \mathrm{k}_{\mathrm{B}}, \mathrm{k}_{\mathrm{S}}$ are the wave-vectors of the three waves, $\mathrm{d}_{\mathrm{eff}}=\chi^{(2)} / 2$ and $\Delta \mathrm{k}=\mathrm{k}_{\mathrm{A}}+\mathrm{k}_{\mathrm{B}}-\mathrm{k}_{\mathrm{S}}$.

For the case of PPLN, the three-wave mixing interaction in (1) was reduced to two nonlinearly coupled equations, where the periodic inversion of the ferroelectric domains was modeled by a periodic function $\mathrm{d}_{\mathrm{eff}}(\mathrm{z})$.

Fig.3 and Fig.4 illustrate the numerical results of SHG obtained for PPLN and KTP, respectively. They report the integrated power $(\mathrm{P})$ of $\mathrm{SH}$ along the transverse domain, normalized by the associated maximum value $\left(\mathrm{P}_{\mathrm{MAX}}\right)$. Both figures confirm the widening of the SHG conversion at high intensities. Moreover, Fig. 3 also confirms the presence of an asymmetry for negative values of $\Delta \mathrm{kL}$ (i.e. for temperatures above the phase-matching condition, where spatial selfdefocusing is expected). In the experiments we noticed a slight shift of the optimal point of conversion efficiency; however the temperature shift was of about $1^{\circ} \mathrm{C}$ and such a shift was within the incertitude of measurement of the temperature.

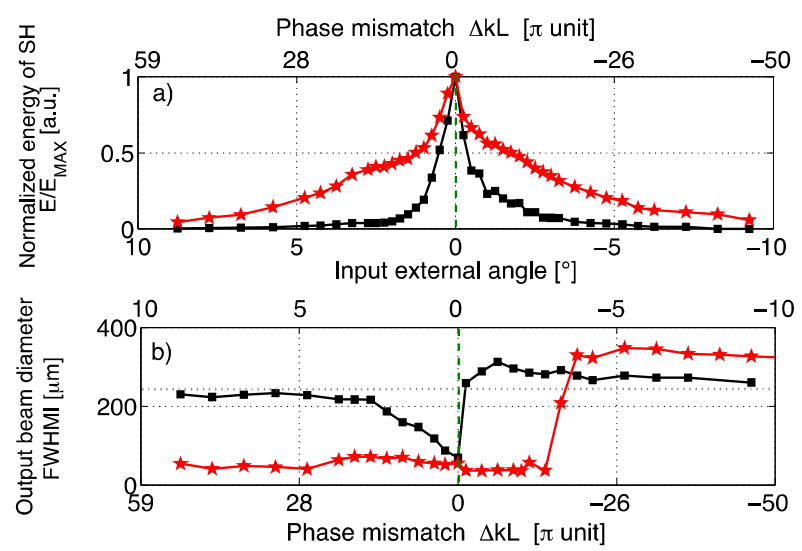

Fig.2. Experimental results of SHG (a) and evolution of output fundamental beam diameter (b) as a function of phase-mismatch $(\Delta \mathrm{kL})$, i.e. input external angle for KTP crystal at low of $0.13 \mathrm{GW} / \mathrm{cm}^{2}$ (black curve) and high of $0.9 \mathrm{GW} / \mathrm{cm}^{2}$ (red curve) pump intensities; phase-matching conditions are represented by green dashed line 


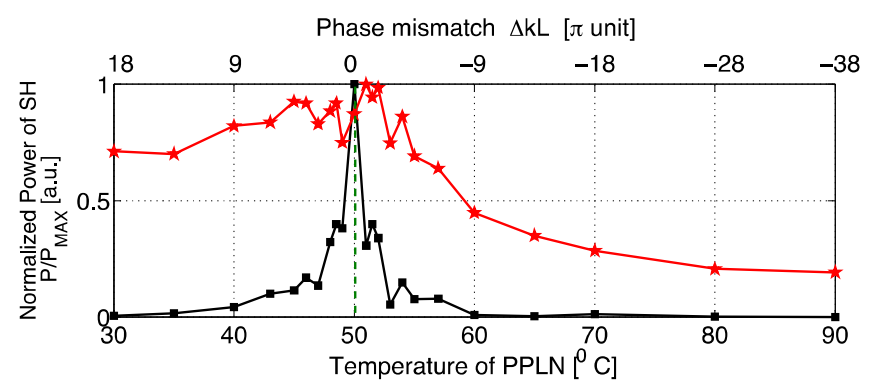

Fig.3. Numerical simulations of SHG as a function of phase-mismatch $(\Delta \mathrm{kL})$, i.e. temperature for PPLN crystal at low of $0.15 \mathrm{GW} / \mathrm{cm}^{2}$ (black curve) and high of $2 \mathrm{GW} / \mathrm{cm}^{2}$ (red curve) pump intensities; $\mathrm{d}_{\mathrm{eff}}=25 \mathrm{pm} / \mathrm{V}$ and phasematching temperature for $\mathrm{SHG}$ is at $50^{\circ} \mathrm{C}$ (green dashed line)

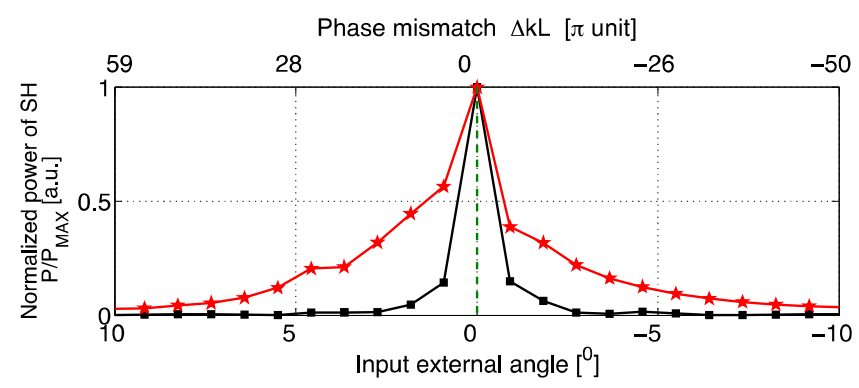

Fig.4. Numerical simulations of SHG as a function of phase-mismatch $(\Delta \mathrm{kL})$, i.e. input external angle for KTP crystal at low of $0.1 \mathrm{GW} / \mathrm{cm}^{2}$ (black curve) and high of $1 \mathrm{GW} / \mathrm{cm}^{2}$ (red curve) pump intensities; phase-matching conditions are represented by green dashed line

\section{CONCLUSIONS}

In this work we studied the efficiency of second harmonic generation and the co-existing effect of spatial beam reshaping in the regime of relatively high pump intensities. We considered two types of bulk quadratic crystals cut for SHG: a PPLN crystal for type 0 QPM and a KTP crystal for type II. We showed that in both crystals the dependence of the energy of the generated second harmonic upon the phase-mismatch values significantly varies at high intensities with respect to the well-known sinc shape. We experimentally demonstrated a significant increase of acceptance bandwidth for SHG, which might be predicted basing on the theoretical description reported in Ref.[3]. We highlighted that, in our experiments, besides such atypical SHG behavior, we observed an unusual beam re-focusing phenomenon, irrespective of the sign of $\Delta \mathrm{kL}$. We believe that such complex and uncommon nonlinear effects may find useful applications to high-energy supercontinuum generation in nonlinear crystals. Our experiments were quantitatively well reproduced by our numerical simulations of SHG.

\section{ACKNOWLEDGMENT}

We acknowledge the financial support from Bpifrance OSEO (Industrial Strategic Innovation Program) and from Région Limousin (C409-SPARC).

\section{REFERENCES}

[1] L. G. Wright, D. N. Christodoulides, and F. W. Wise, "Controllable spatiotemporal nonlinear effects in multimode fibres," Nat. Photon., vol. 9, pp. 306-310, April 2015.

[2] X. Qi, I. L. Garanovich, A. A. Sukhorukov, W. Krolikowski, A. Mitchell, G. Zhang, D. N. Neshev, Y. S. Kivshar, "Polychromatic solitons and symmetry breaking in curved waveguide arrays," Opt. Lett., vol. 35, pp.1371-1373, 2010.

[3] S. Trillo, S. Wabnitz, R. Chisari, G. Cappellini, "Two-wave mixing in a quadratic nonlinear medium: bifurcations, spatial instabilities, and chaos," Opt. Lett., vol. 17, pp.637-639, 1992

[4] R. W. Boyd, "Nonlinear Optics", Third Edition, Rochester, New York, 2007

[5] P. Di Trapani, A. Bramati, S. Minardi, W. Chinaglia, C. Conti, S. Trillo, J. Kilius, G. Valiulis, "Focusing versus Defocusing nonlinearities due to parametric wave mixing," Phys. Rev. Lett., vol. 87, pp.183902-1-4, 2001

[6] C. Conti, S. Trillo, P. Di Trapani, J. Kilius, A. Bramati, S. Minardi, W. Chinaglia, G. Valiulis, "Effective lensing effects in parametric frequency conversion," J. Opt. Soc. Am. B, vol. 19, pp.852-859, 2002

[7] W. E. Torruellas, Z. Wang, E. W. Van Stryland, G. I. Stegeman L. Torner, C. R. Menyuk, "Observation of two-dimensional spatial solitary waves in a quadratic medium," Phys. Rev. Lett., vol. 74, pp.5036-5040, 1995.

[8] B. Bourliaguet, V. Couderc. A. Barthélémy, G. W. Ross, P. G. R. Smith, D. C. Hanna, C. De Angelis, "Observation of quadratic spatial solitons in periodically poled lithium niobate," Opt. Lett., vol. 24, pp.1410-1412, 1999.

[9] A. V. Buryak, Y. S. Kivshar, and S. Trillo,"Parametric spatial solitary wave due to type II second-harmonic generation", Journ. Opt. Soc. Am B, vol.14, pp. 3110-3118, November 1997. 